\title{
Association between Hyperuricemia and Ischemic Stroke: A Case-Control Study
}

\author{
Tooba Fatima ${ }^{1}$, Sadaf Iftikhar ${ }^{2}$ and Irshad Hussain Qureshi ${ }^{1}$ \\ ${ }^{1}$ Department of Medicine, King Edward Medical University / Mayo Hospital, Lahore, Pakistan \\ ${ }^{2}$ Department of Neurology, King Edward Medical University / Mayo Hospital, Lahore, Pakistan
}

\begin{abstract}
Objective: To find the association between hyperuricemia and ischemic stroke.

Study Design: Case-control study.

Place and Duration of Study: Departments of Medicine and Neurology, Mayo Hospital, Lahore, from October 2015 to September 2016.

Methodology: A total of 100 patients were recruited in the study. Fifty cases were of acute ischemic stroke and fifty were age- and gender-matched healthy controls. Serum uric acid level was estimted by photometry method at the time of admission. Hyperuricemia was defined as the serum uric acid level of more than $6 \mathrm{mg} / \mathrm{dl}$.

Results: Mean serum uric acid levels in cases and controls were $5.996 \pm 1.99 \mathrm{mg} / \mathrm{dl}$ and $5.042 \pm 0.91 \mathrm{mg} / \mathrm{dl}$, respectively. Hyperuricemia was present in $23(46 \%)$ patients with ischemic stroke and in $10(20 \%)$ controls. Among ischemic stroke patients, the frequency of hyperuricemia was significantly higher than in controls ( $p$-value $=0.006$, Odds Ratio $(O R)=3.41$.)

Conclusion: Serum uric acid was found significantly elevated in ischemic stroke patients versus controls with 3.41 times more risk of having hyperuricemia in ischemic stroke patients.
\end{abstract}

Key Words: Hyperuricemia, Ischemic stroke, Serum uric acid (SUA).

How to cite this article: Fatima T, Iftikhar S, Qureshi IH. Association between Hyperuricemia and Ischemic Stroke: A Case-Control Study. J Coll Physicians Surg Pak 2020; 30(08):853-856.

\section{INTRODUCTION}

Stroke is a leading cause of morbidity and mortality inflicting more than half of stroke patients aged $\geq 65$ years, yet it is a preventable disease. ${ }^{1}$ Hypertension, hypercholesterolemia, diabetes mellitus, atrial fibrillation, carotid artery stenosis and smoking are definite risk factors for stroke ${ }^{2}$. Current evidence shows that the identification of nontraditional risk factors for stroke is increasing; which include metabolic syndrome, obesity, sleep apnea syndrome, chronic inflammation, chronic renal disease, and nutrition. Elevated serum uric acid, i.e. hyperuricemia, is quite a common finding in patients with metabolic syndrome, obesity, hypertension, diabetes mellitus, chronic renal disease and cardiovascular events. ${ }^{3}$ Although, serum uric acid (SUA) is one of the potent antioxidants in plasma and seemingly having a neuroprotective role in the animal models; the results from human studies are diverse and controversial.

Correspondence to: Dr. Tooba Fatima, East Medical Ward, King Edward Medical University / Mayo Hospital, Lahore, Pakistan

E-mail:dr tooba@hotmail.com

Received: March 15, 2019; Revised: December 02, 2019;

Accepted: February 19, 2020

DOI: https://doi.org/10.29271/jcpsp.2020.08.853
On one hand, it is shown that the low SUA level strongly and independently predicts poor short- and long-term outcomes after ischemic stroke, and independently correlates with more severity, unfavourable evolution of stroke, and vascular events following stroke. ${ }^{4,5,6}$ Whereas, on the other hand, in the Mexican multi-centre study, low SUA level was found associated with quite good short-term outcome in ischemic stroke patients. ${ }^{7}$ In a study from the Netherlands, Miedemal et al found no association between SUA levels and both short- and long-term outcomes in ischemic stroke. ${ }^{8}$ Recently, Wang, et al ${ }^{9}$ reported hyperuricemia as independent predictor of poor in-hospital outcome in diabetic ischemic stroke patients aged $\leq 75$ years; and found no association between hyperuricemia and prognosis of stroke in both diabetic and non-diabetic patients. ${ }^{9}$ Contrary to this, a study by Wang YF, et al. ${ }^{10}$ showed that hyperuricemia is a significant protective factor in males and in a large artery atherosclerosis subtype of ischemic stroke patients. ${ }^{10}$

Prevalence of hyperuricemia in ischemic stroke has been studied world-wide and shown variable findings. Mapoure et al. demonstrated hyperuricemia in more than half $(52.3 \%)$ Black African patients with ischemic stroke. ${ }^{11}$ In an Indian study, ${ }^{12}$ the SUA level was found significantly elevated $(>6 \mathrm{mg} / \mathrm{dl}$ ) in ischemic stroke patients $54.9 \%$ versus $24.7 \%$ in controls $(p<0.05) .{ }^{14}$ In another South Indian study, 30\% of acute ischemic stroke patients were hyperuricemic. ${ }^{13}$ An Iranian study reported $13.0 \%$ ischemic stroke patients versus $10.7 \%$ 
controls having hyperuricemia; and no significant relationship was found between ischemic stroke and elevated serum uric acid level $(p>0.05) .{ }^{14}$ In another Iranian study by Mehrpour et al, $47.3 \%$ of ischemic stroke patients were found to have hyperuricemia. ${ }^{15}$ Keeping these diverse results in view, this study was conducted to determine the association between hyperuricemia and acute ischemic stroke patients since no such data has been published so far from Pakistan.

\section{METHODOLOGY}

This case-control study was conducted in the Department of Medicine, Mayo Hospital, Lahore, Pakistan from October 2015 toSeptember 2016. The sample size was 100 subjects and calculated with $80 \%$ power of test, $5 \%$ level of significance, and taking an expected percentage of hyperuricemia as $54.9 \%$ in ischemic stroke patients and $24.7 \%$ in controls. ${ }^{12}$ After getting approval from the Institutional Review Board, King Edward Medical University / Mayo Hospital, Lahore, 50 patients of acute ischemic stroke of either gender, aged between 40-75 years presenting within 12 hours of its onset and 50 age- and gendermatched healthy controls, were recruited. All ischemic stroke patients were examined by a neurologist and were diagnosed as per American Heart Association/American Stroke Association criteria ${ }^{16}$. The controls had no clinical evidence of any cerebrovascular disease. Subjects with liver or renal disease, diabetes mellitus, cardiovascular disease, thyroid dysfunction and inflammatory bowel disease, were excluded. None of the subjects had a history of alcohol consumption or was receiving any lipid-lowering drugs (e.g., the statins) or any medication that affects the level of uric acid (e.g., corticosteroids, allopurinol).

Serum uric acid level along with other routine labs, were measured at the time of admission. Hyperuricemia was defined as the serum uric acid level of more than $6 \mathrm{mg} / \mathrm{dl} .{ }^{9}$ Patients' data was collected on the pre-designed proforma after taking written informed consent either from the patients or their spouses/relatives, if patienthad reduced level of consciousness or aphasia.

Data was analysed by using statistical package for the social sciences (SPSS) version 20. Quantitative variables like age and serum uric acid level were presented as means and standard deviations. Qualitative variables like gender and hyperuricemia were presented as frequencies and percentages. Student t-test was applied to calculate p-value for serum uric acid among ischemic stroke patients and controls. Chi-Square test was applied to calculate $p$-value and odds ratio (OR) to look for the association of hyperuricemia with ischemic stroke and controls. Data was stratified for age $(40-60,61-80$ years) and gender (male/female). Post-stratification, adjusted OR was calculated. The $p$-value $<0.05$ and $O R>1$ were considered statistically significant.

\section{RESULTS}

The mean age of the patients with ischemic stroke was 60.82 \pm 9.30 years, the minimum age was 40 years and maximum was 75 years; whereas, among controls, the mean age was 55.20 \pm 9.75 years the minimum age was 41 years and maximum was 75 years. Among ischemic stroke patients, there were 25 (50\%) males and 25 (50\%) females; whereas, among controls, there were 30(60\%) males and 20 (40\%) females.

The mean SUA level was $5.996 \pm 1.99 \mathrm{mg} / \mathrm{dl}$ in ischemic stroke patients, while the mean SUA level was $5.042 \pm 0.91 \mathrm{mg} / \mathrm{dl}$ in controls showing statistical significance $(p=0.003)$.

Frequency of hyperuricemia among ischemic stroke patients was 46\% (23/50); and among controls, was 20\% (10/50). The patients with ischemic stroke are 3.41 times at more risk for having hyperuricemia as compared to controls. Thus, hyperuricemia is a significant risk factor for ischemic stroke $(p=$ 0.006, Tablel).

Table I: Association between hyperuricemia and ischemic stroke.

\begin{tabular}{|c|c|c|c|}
\hline & $\begin{array}{l}\text { Ischemic } \\
\text { Stroke }\end{array}$ & Control & Total \\
\hline $\begin{array}{l}\text { Hyperuricemia } \\
\text { Yes } \\
\text { No }\end{array}$ & $\begin{array}{l}23(46 \%) \\
27(54 \%)\end{array}$ & $\begin{array}{l}10(20 \%) \\
40(80 \%)\end{array}$ & $\begin{array}{l}33 \\
67\end{array}$ \\
\hline Total & 50 & 50 & 100 \\
\hline
\end{tabular}

There was a significant association between hyperuricemia and ischemic stroke in the age group of $40-60$ years $(p=0.015)$. There was also a 4.06 times more risk of having hyperuricemia in ischemic stroke than controls. Whereas, in the age group of 61-80 years, there was no significant association between hyperuricemia and ischemic stroke. There was also a 2.4 times more risk of having hyperuricemia in ischemic stroke. There was no significant association between hyperuricemia and ischemic stroke among males and the odds of developing hyperuricemia was 2.58 times more than controls; whereas, among females, there was a significant association between hyperuricemia and ischemic stroke $(p=0.020)$ and there was 5.23 times more odds of developing hyperuricemia in females with ischemic stroke as compared to the controls (Tablell).

\section{DISCUSSION}

In this study, serum uric acid was found to be significantly elevated in acute ischemic stroke patients versus controls; also the risk of having hyperuricemia was found 3.41 times more in ischemic stroke patients. Several experimental studies have demonstrated uric acid to be a potent antioxidant and neuroprotectant, and reported hyperuricemia as a compensatory mechanism to counteract free radical injury. Chamorro et al. found a $12 \%$ increase in the odds of good functional outcome for each milligram per deciliter ( $\mathrm{mg} / \mathrm{dl}$ ) increase of serum uric acid in patients with ischemic stroke. ${ }^{17}$ In contrast, hyperuricemia has been linked to various pro-atherogenic processes, i.e. increased oxidative stress or damage, smooth muscle proliferation in the vessels and a casca de of leukocytic activation. Arévalo-Lorido et al recently demonstrated positive correlation between SUA levels and intima to media thickness in the carotid arteries and found greater atherosclerotic carotid artery disease in ischemic stroke patients with the highest levels of serum uric acid $(p<0.005){ }^{18}$ 
Table II: Association between hyperuricemia and ischemic stroke stratified for age and gender.

\begin{tabular}{|c|c|c|c|c|c|c|}
\hline Age (years) & Hyperuricemia & Ischemic stroke & Control & Chi-square test & p-value & Odd's ratio \\
\hline \multirow{2}{*}{$40-60$} & Yes & $11(47.8 \%)$ & $7(18.4 \%)$ & \multirow{2}{*}{5.95} & \multirow{2}{*}{0.015} & \multirow{2}{*}{4.06} \\
\hline & No & $12(52.2 \%)$ & $31(81.6 \%)$ & & & \\
\hline \multirow{2}{*}{$61-80$} & Yes & $12(44.4 \%)$ & $3(25 \%)$ & \multirow{2}{*}{1.32} & \multirow{2}{*}{0.249} & \multirow{2}{*}{2.4} \\
\hline & No & $15(55.6 \%)$ & $9(75 \%)$ & & & \\
\hline \multicolumn{7}{|l|}{ Gender } \\
\hline \multirow{2}{*}{ Male } & Yes & $11(44 \%)$ & $7(23.3 \%)$ & \multirow{2}{*}{2.64} & \multirow{2}{*}{0.104} & \multirow{2}{*}{2.58} \\
\hline & No & $14(56 \%)$ & $23(76.7 \%)$ & & & \\
\hline \multirow{2}{*}{ Female } & Yes & $12(48 \%)$ & $3(15 \%)$ & \multirow{2}{*}{5.44} & \multirow{2}{*}{0.020} & \multirow{2}{*}{5.23} \\
\hline & No & $13(52 \%)$ & $17(85 \%)$ & & & \\
\hline
\end{tabular}

In the present study, it was found that hyperuricemia i.e., SUA level more than $6 \mathrm{mg} / \mathrm{dl}$, in $46 \%$ ischemic stroke patients versus $20 \%$ controls. Mean SUA level in patients and controls was $5.996 \pm 1.99 \mathrm{mg} / \mathrm{dl}$ and $5.042 \pm 0.91 \mathrm{mg} / \mathrm{dl}$ $p=0.003$ ) respectively. Also, frequency of hyperuricemia was significantly higher in ischemic stroke patients than in controls, i.e., $=0.006$ and OR 3.41, which means there was 3.41 times more risk of having hyperuricemia in ischemic stroke patients than in controls. Cases who were in the age range of 40-60 years, the odds of having hyperuricemia was 4.06; and for those in the age range of $61-80$ years, it was 2.4. These findings are quite comparable with the results of a South Indian study, ${ }^{12}$ in which the SUA levels were found to be significantly elevated, $54.9 \%$ in stroke patients versus $24.7 \%$ in controls. Mean SUA level in cases and controls was $6.14 \pm 1.68 \mathrm{mg} / \mathrm{dl}$ and $4.12 \pm 2.20 \mathrm{mg} / \mathrm{dl}$, respectively, $\mathrm{p}$ value $<0.001$ and odd's ratio of 3.70 , which meant there was 3.70 times more chances of having hyperuricemia in ischemic stroke patients versus controls. However, in another South Indian study, ${ }^{13}$ mean serum uric acid level in acute ischemic stroke patients was found to be $5.5 \pm 1.7$ $\mathrm{mg} / \mathrm{dl}$, and $30 \%$ ischemic stroke patients were hyperuricemic. Moreover, SUA levels were significantly higher in females than males and were found predominant in the age range of 56-70 years. Their findings were consistent with the present results as well. They concluded that hyperuricemia and concomitant dyslipidemia can be considered as the risk factor for acute ischemic stroke. A cross-sectional study, from Iran reported mean (5.94 $\pm 1.70 \mathrm{mg} / \mathrm{dl}$ ) SUA level in ischemic stroke patients in $47.3 \%$ patients with hyperuricemia. ${ }^{15}$ They also concluded that due to high frequency of hyperuricemia and accompanying increase in triglyceride and low-density lipoprotein cholesterol levels; hyperuricemia can be considered as a risk factor for acute ischemic stroke. Another Iranian study demonstrated $\mathbf{1 3 . 0 \%}$ ischemic stroke patients versus $10.7 \%$ controls having hyperuricemia and no significant relationship was found between ischemic stroke and elevated serum uric acid level $(p>0.05)$ that was contrary to the present findings. ${ }^{14}$

In a Bangladeshi case-control study, ${ }^{19}$ the mean SUA levels of ischemic stroke patients and controls were as follows: 4.94 \pm 1.76 and $3.72 \pm 1.09$, respectively; $(p<0.05) .23 .3 \%$ of patients had an abnormal SUA level, while $6.7 \%$ of controls had abnormal SUA level. Whereas, in this study, mean SUA was $5.996 \pm 1.99 \mathrm{mg} / \mathrm{dl}$ in ischemic stroke patients, while mean SUA was $5.042 \pm 0.91 \mathrm{mg} / \mathrm{dl}$ in controls $(p=0.003)$. Mapoure et al. found hyperuricemia in more than half (52.3\%) Black African patients with ischemic stroke, ${ }^{11}$ that was higher than the frequency of hyperuricemia seen in this study population. Sarfo, et al. reported frequency of hyperuricemia among ischemic stroke patients in Ghana as $46.3 \%,{ }^{20}$ that is quite comparable with this finding, but their number of stroke patients with hyperuricemia increased with increasing age, whichis not observed in this study. Here, a significant association was found between hyperuricemia and ischemic stroke in age group of $40-60$ years $(p=0.015)$; whereas, in the age group of 61-80 years, there was no significant association found between hyperuricemia and ischemic stroke ( $p=0.249)$. These differences could be due to diversity in ethnicity, geography, diet or lifestyle modifications.

This study has a few limitations, i.e. it is a hospital-based, single-centre study. Secondly, it was conducted on those ischemic stroke patients who belonged to low socio-economic status and had heterogeneous risk factor profiles; so these results cannot be applied to the general population.

\section{CONCLUSION}

The frequency of hyperuricemia was found significantly higher $(46 \%)$ in ischemic stroke patients. Moreover, the risk of having hyperuricemia is 3.41 times more in patients with ischemic stroke. Therefore, hyperuricemia may be investigated as other risk factors for ischemic stroke. However, more research is needed before routine assessment of serum uric acid levels can be recommended.

\section{DISCLOSURE:}

The manuscript is the original study of the first author for her FCPS Part-II thesis work.

\section{ETHICAL APPROVAL:}

Ethical approval was obtained prior to initiation of this research work from the Institutional Review Board of King Edward Medical University / Mayo Hospital, Lahore.

\section{PATIENTS' CONSENT:}

Informed consents were obtained from all patients who participated in this study to publish the data. 


\section{CONFLICT OF INTEREST:}

The authors declared no conflict of interest.

\section{AUTHORS' CONTRIBUTION:}

TF: Data acquisition and analysis, interpretation, drafting, integrity of the work.

SI: Conception and design, interpretation, critical appraisal, revision and final approval.

IHQ: Critical appraisal and final approval.

\section{REFERENCES}

1. Benjamin EJ, Blaha MJ, Chiuve SE, Cushman M, Das SR, Deo $R$, et al. Heart disease and stroke statistics-2017 update: A report from the American Heart Association. Circulation 2017; 135(10): 229-445.

2. Aquil N, Begum I, Ahmed A, Vohra EA, Soomro BA. Risk factors in various subtypes of ischemic stroke according to TOAST criteria. J Coll Physicians Surg Pak 2011; 21(5): 280-3.

3. Soltani Z, Rasheed K, Kapusta DR, Reisin E. Potential role of uric acid in metabolic syndrome, hypertension, kidney injury, and cardiovascular diseases: Is it time for reappraisal? Curr Hypertens Rep 2013; 15(3):175-81.

4. Wu H, Jia Q, Liu G, Liu L, Pu Y, Zhao X, et al. Decreased uric acid levels correlate with poor outcomes in acute ischemic stroke patients, but not in cerebral hemorrhage patients. J Stroke Cerebrovasc Dis 2014; 23(3):469-75.

5. Brouns R, Wauters A, Van De Vijver G, De Surgeloose D, Sheorajpanday $\mathrm{R}$, De Deyn PP. Decrease in uric acid in acute ischemic stroke correlates with stroke severity, evolution and outcome. Clin Chem Lab Med 2010; 48(3):383-90.

6. Wu S, Pan Y, Zhang N, Jun WY, Wang C. Lower serum uric acid level strongly predict short-term poor functional outcome in acute stroke with normoglycaemia: A cohort study in China. BMC Neurol 2017; 17(1):21.

7. Chiquete E, Ruiz-Sandoval JL, Murillo-Bonilla LM, Arauz A, Orozco-Valera, Ochoa-Guzmán A, et al. Serum uric acid and outcome after acute ischemic stroke: Premier study. Cerebrovasc Dis 2013; 35(2):168-74.

8. Miedema I, Uyttenboogaart M, Koch M, Kremer B, de Keyser J, Luijckx GJ. Lack of association between serum uric acid levels and outcome in acute ischemic stroke. J Neurol Sci 2012; 319(1-2):51-5.

9. Wang $P$, Li X, He C, Zhai $Y$, Sun H, Zhang $Y$, et al. Hyper- uricemia and prognosis of acute ischemic stroke in diabetic patients. Neurological Research 2019; 41(3): 250-6.

10. Wang YF, Li JX, Sun XS, Lai R, Sheng WL. High serum uric acid levels are a protective factor against unfavourable neurological functional outcome in patients with ischemic stroke. J Int Med Res 2018; 46(5):1826-38.

11. Mapoure YN, Ayeah CM, Ba H, Hentchoya R, Luma HN. The prognostic value of serum uric acid in the acute phase of ischemic stroke in Black Africans. J Stroke Cerebrovasc Dis 2018; 27(3):783-92.

12. Koppula R, Kaul S, Venkateswar Rao A, Jyothy A, Munshi A. Association of serum uric acid level with ischemic stroke, stroke subtypes and clinical outcome. Neurology Asia 2013; 18(4):349-53.

13. Arora T, Mantur PG, Bidri RC, Mulimani MS. Serum uric acid levels and serum lipid levels in patients with ischemic cerebrovascular accident. J Assoc Physicians India 2018; 66:349-54.

14. Iranmanesh F, Sheykholeslami NZ, Gadari F, Ahmady J. Acute ischemic non-embolic stroke and serum level of uric acid. Iran J Neurol 2012; 11(1):1-5.

15. Mehrpour M, Khuzan M, Najimi N, Motamed MR, Fereshtehnejad SM. Serum uric acid level in acute stroke patients. Med J Islam Repub Iran 2012; 26(2):66-72.

16. Sacco RL, Kasner SE, Broderick JP, Caplan LR, Connors JJ, Culebras $A$, et al. An updated definition of stroke for the 21st Century. A statement for healthcare professionals from the american heart association/american stroke association. Stroke 2013; 44 (7): 2064-89.

17. Chamorro Á, Obach V, Cervera Á, Revilla M, Deulofeu R, Aponte JH. Prognostic significance of uric acid serum concentration in patients with acute ischemic stroke. Stroke 2002; 33(4):1048-52.

18. Arévalo-Lorido JC, Carretero-Gómez J, Robles Pérez-Monteoliva NR. Association between serum uric acid and carotid disease in patients with atherosclerotic acute ischemic stroke. Vascular 2019; 27(1):19-26.

19. Khalil MI, Islam MJ, Ullah MA, Khan RK, Munira S, Haque MA, et al. Association of serum uric acid with ischemic stroke. Mymensingh Med J 2013; 22(2):325-30.

20. Sarfo F, Akassi J, Antwi N, Obese V, Adamu S, Akpalu A, et al. Highly prevalent hyperuricaemia is associated with adverse clinical outcomes among Ghanaian stroke patients: An observational prospective study. Ghana Med J 2015; 49(3):165-72. 